\title{
Novel ethyl 1,5-disubstituted-1H-pyrazole-3-carboxylates as a new class of antimicrobial agents
}

\author{
AWWAD A. RADWAN ${ }^{1,2 *}$ \\ MOSTAFA M. GHORAB 3 ,4* \\ MANSOUR S. ALSAID ${ }^{3}$ \\ FARES K. ALANAZI ${ }^{1}$ \\ ${ }^{1}$ Department of Pharmaceutics \\ College of Pharmacy \\ King Saud University, P.O. Box 2457 \\ Riyadh 11451, Saudi Arabia \\ ${ }^{2}$ Department of Pharmaceutical \\ Organic Chemistry \\ Faculty of Pharmacy \\ Assiut University, Assiut-71527, Egypt \\ ${ }^{3}$ Department of Pharmacognosy \\ College of Pharmacy \\ King Saud University, Riyadh \\ P.O. 2457, Riyadh 11451, Saudi Arabia \\ ${ }^{4}$ Department of Drug Radiation \\ Research, National Centre For \\ Radiation Research and Technology \\ Atomic Energy Authority \\ P.O. Box 29, Nasr City, Cairo, Egypt
}

\begin{abstract}
A series of pyrazole derivatives 9-22 were designed and synthesized. All the newly synthesized compounds were assayed for their antimicrobial activity against the Grampositive bacteria Staphyllococcus aureus and Bacillius subtilis and the Gram-negative bacteria Escherichia coli, Pseudomonas aeruginosa, in addition to the fungi organisms, Candida albicans, C. parapsilosis and C. tropicalis. Ethyl 5-(2,5-dimethylthiophen-3-yl)-1-phenyl-1H-pyrazole-3-carboxylate (21) $\left(\mathrm{MIC}_{\text {E.coli }}=0.038 \mu \mathrm{mol} \mathrm{mL} \mathrm{mL}^{-1}, \mathrm{MIC}_{P \text {. aerug. }}=0.067 \mu \mathrm{mol} \mathrm{mL}^{-1}\right)$ is nearly as active as ampicillin $(\mathrm{MIC}=0.033$ and 0.067 $\left.\mu \mathrm{mol} \mathrm{mL} \mathrm{L}^{-1}\right)$, respectively. Ethyl5-(4-bromo-2-chlorophenyl)1-phenyl-1H-pyrazole-3-carboxylate $(\mathbf{1 6}) \quad(\mathrm{MIC}=0.015$ $\left.\mu \mathrm{mol} \mathrm{mL} \mathrm{m}^{-1}\right)$ is more active than fluconazole $\left(0.020 \mu \mathrm{mol} \mathrm{mL} \mathrm{mL}^{-1}\right)$ as a reference drug against $C$. parapsilosis.
\end{abstract}

Keywords: pyrazole-3-carboxylates, antimicrobial activity

Accepted May 15, 2014

Nitrogen heterocycles are of special interest because they constitute an important class of natural and non-natural products, many of which exhibit useful biological activities. Pyrazoles and their derivatives exhibit antimicrobial (1-3), anticancer (4), antiinflammatory (5) radioprotective (6), anticonvulsant (7) and antimalarial (8) activities. In the light of these facts, and as a continuation of our efforts towards synthesizing biologically active heterocyclic compounds (9-18), we aimed to prepare new derivatives of pyrazoles. The compounds were designed with the aim of exploring their antimicrobial activity and to study their structure-activity relationship.

\footnotetext{
*Correspondence; e-mail address: mmsghorab@yahoo.com; dhna_2001@hotmail.com
} 


\section{EXPERIMENTAL}

Melting points were uncorrected and determined on an electrothermal melting point apparatus (Stuart Scientific, UK). Precoated silica gel plates (Kieselgel 0.25 mm, 60G F254, Merck, Germany) were used for thin layer chromatography. Developing solvent system was composed of chloroform/methanol (8:2) and the spots were detected under UV. IR spectra (KBr discs) were recorded on a FT-IR spectrophotometer (Perkin Elmer, USA). NMR spectra were scanned on a NMR spectrophotometer (Bruker, Switzerland) operating at $500 \mathrm{MHz}$ for ${ }^{1} \mathrm{H}$ - and $125.76 \mathrm{MHz}$ for ${ }^{13} \mathrm{C}$ NMR. Chemical shifts are expressed in $\delta$-values (ppm) relative to TMS as an internal standard, using DMSO- $d_{6}$ as a solvent. Elemental analyses were done on a model 2400 CHNSO analyzer (Perkin Elmer, USA). All reagents were purchased from Sigma (USA). Compounds 1 and $\mathbf{9}$ were prepared according to the reported methods $(19,20)$.

\section{Synthesis of ethyl 4-(substituted phenyl)-2-hydroxy-4-oxobut-2-enoates (1-8)}

Ethanol (100 mL) was converted to sodium ethoxide by portionwise addition of sodium $(0.46 \mathrm{~g}, 0.02 \mathrm{~mol})$ before a solution of diethyl oxalate $(2.92 \mathrm{~g}, 0.02 \mathrm{~mol})$ and acetophenone derivative, namely, 4-chloroacetophenone, 3,4-dimethoxyacetophenone, 2,5-dimethoxyacetophenone, 2,5-dichloroacetophenone, 2-chloro-4-bromoacetophenone, 1-(naphthalene1-yl)ethanone, 1-(thiophen-2-yl)ethanone and/or 1-(2,5-dimethylthiophene-3-yl)ethanone $(0.01 \mathrm{~mol})$ in ethanol $(50 \mathrm{~mL})$ was added dropwise at $50{ }^{\circ} \mathrm{C}$. The reaction mixture was heated under reflux for 2-3 h. After cooling the solvent was removed and the residue was taken up in water $(200 \mathrm{~mL})$ and acidified with concentrated $\mathrm{HCl}(1 \mathrm{~mL})$. The aqueous mixture was extracted with diethyl ether $(3 \times 150 \mathrm{~mL})$. The combined extracts were washed with brine $(100 \mathrm{~mL})$, dried $\left(\mathrm{MgSO}_{4}\right)$, and concentrated. The obtained solid was recrystallized from methanol to give compounds $1-8$, respectively.

\section{Synthesis of ethyl 1,5-disubstituted-1H-pyrazole-3-carboxylates (9-22)}

A mixture of compound 1-8 $(0.01 \mathrm{~mol})$ with arylhydrazine derivative $(0.012 \mathrm{~mol})$ in ethanol $(50 \mathrm{~mL})$ was refluxed for $8 \mathrm{~h}$. The reaction mixture was cooled and poured into ice water. The obtained solid was recrystallized from dioxane to give compounds $\mathbf{9 - 2 2}$, respectively.

Physico-chemical, analytical and spectral data are displayed in Tables I and II. Synthetic pathway is presented in Scheme 1 .

Staphyloccocus aureus (NCTC-7447) and Bacillius subtilis (NCTC 1040) and Gram-negative bacteria E. coli (NCTC 10416), and Pseudomonas aeruginosa (ATCC 10145), in addition to the fungi organisms, Candida albicans (ATCC-10231), Candida parapsilosis (ATCC-22019) and Candida tropicalis (ATCC-66029) were tested. All microorganisms were purchased from American Type Culture Collection (Manassas, USA).

The antimicrobial activity screening of the newly synthesized compounds (9-22) was undertaken using the agar cup diffusion ( $8 \mathrm{~mm}$ diameter) assay I (21). The agar media were inoculated with test organisms and a solution of test compound $100 \mu \mathrm{g} \mathrm{mL}^{-1}$ in DMSO. Ampicillin (100 and $\left.50 \mu \mathrm{g} \mathrm{mL}^{-1}\right)$, and fluconazole $\left(2\right.$ and $1 \mu \mathrm{g} \mathrm{mL}^{-1}$ ) were used as reference drugs for antibacterial and antifungal activity, respectively. The zones of inhibition were measured after $24 \mathrm{~h}$ of incubation. 
For determination of minimum inhibitory concentration (MIC) (21) by serial plate dilution method, five milligrams of each test compounds were dissolved in $1 \mathrm{~mL}$ of dimethylsulfoxide (DMSO) separately to prepare stock solution. From stock solution serial dilutions were prepared. The plates were incubated at $37^{\circ} \mathrm{C}$ for $24 \mathrm{~h}$. MIC is the lowest concentration $\left(\mu \mathrm{mol} \mathrm{mL} \mathrm{mL}^{-1}\right)$ of the test compound that resulted in no visible growth on the plates. DMSO was used as a solvent control to ensure that solvent had no effect on bacterial growth. The results of the antimicrobial activities are summarized in Table III.

\section{RESULTS AND DISCUSSION}

\section{Chemistry}

$\beta$-Diketones 1-8 were prepared by Claisen reaction between acetophenone derivatives and diethyl oxalate (Scheme 1). The synthesis of 1,5-diarylpyrazoles 9-22 was done by regioselective cyclization of arylhydrazines with $\beta$-diketones 1-8 in refluxing ethanol. Tables I and II show the physicochemical and the spectral data. The physicohemical data of compounds 1 and 9 are in accordance with those reported in the literature $(19,20)$. The structures of compounds 2-8 and 10-22 were confirmed by elemental analyses, IR, ${ }^{1} \mathrm{H}$ NMR and ${ }^{13} \mathrm{C}$ NMR spectral data. IR spectra of compounds $2-8$ showed the presence of characteristic bands for $\mathrm{OH}\left(3462-3421 \mathrm{~cm}^{-1}\right), 2 \mathrm{C}=\mathrm{O}\left(1751-1708\right.$ and $\left.1685-1625 \mathrm{~cm}^{-1}\right) .{ }^{1} \mathrm{H}$ NMR spectra of compounds 2-8 revealed the presence of a triplet at 1.2-1.3 ppm assigned to $\mathrm{CH}_{3}$ ester and a quartet at 4.1-4.4 ppm due to $\mathrm{CH}_{2}$ ester. Mass spectrum of compound 2 showed

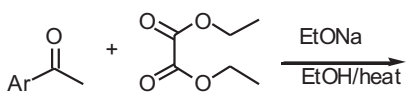<smiles>CCOC(=O)/C(O)=C/C(=O)Br</smiles>

$1-8$
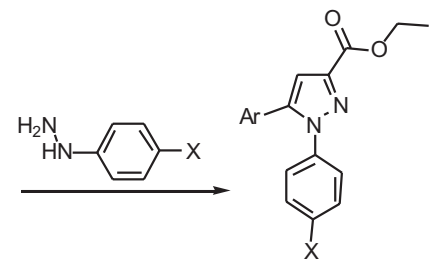

9-22

\begin{tabular}{c|l} 
Compd. & Ar \\
\hline $\mathbf{1}$ & $\mathrm{C}_{6} \mathrm{H}_{4} \mathrm{Cl}-4$ \\
2 & $\mathrm{C}_{6} \mathrm{H}_{3}\left(\mathrm{OCH}_{3}\right)_{2}-3,4$ \\
3 & $\mathrm{C}_{6} \mathrm{H}_{3}\left(\mathrm{OCH}_{3}\right)_{2}-2,5$ \\
4 & $\mathrm{C}_{6} \mathrm{H}_{3} \mathrm{Cl}_{2}-2,5$ \\
5 & $\mathrm{C}_{6} \mathrm{H}_{3} \mathrm{Cl}-2, \mathrm{Br}-4$ \\
$\mathbf{6}$ & 1-naphthyl \\
7 & 2-thienyl \\
8 & 3-thienyl- $\left(\mathrm{CH}_{3}\right)_{2}-2,5$
\end{tabular}

\begin{tabular}{c|l|l} 
Compd. & Ar & $\mathrm{X}$ \\
\hline $\mathbf{9}$ & $\mathrm{C}_{6} \mathrm{H}_{4} \mathrm{Cl}-4$ & $\mathrm{H}$ \\
$\mathbf{1 0}$ & $\mathrm{C}_{6} \mathrm{H}_{4} \mathrm{Cl}-4$ & $\mathrm{Br}$ \\
$\mathbf{1 1}$ & $\mathrm{C}_{6} \mathrm{H}_{3}\left(\mathrm{OCH}_{3}\right)_{2}-3,4$ & $\mathrm{H}$ \\
$\mathbf{1 2}$ & $\mathrm{C}_{6} \mathrm{H}_{3}\left(\mathrm{OCH}_{3}\right)_{2}-3,4$ & $\mathrm{Br}$ \\
$\mathbf{1 3}$ & $\mathrm{C}_{6} \mathrm{H}_{3}\left(\mathrm{OCH}_{3}\right)_{2}-2,5$ & $\mathrm{H}$ \\
$\mathbf{1 4}$ & $\mathrm{C}_{6} \mathrm{H}_{3}\left(\mathrm{OCH}_{3}\right)_{2}-2,5$ & $\mathrm{Br}$ \\
$\mathbf{1 5}$ & $\mathrm{C}_{6} \mathrm{H}_{3} \mathrm{Cl}_{2}-2,5$ & $\mathrm{H}$ \\
$\mathbf{1 6}$ & $\mathrm{C}_{6} \mathrm{H}_{3} \mathrm{Cl}-2 ; \mathrm{Br}-4$ & $\mathrm{H}$ \\
$\mathbf{1 7}$ & $\mathrm{C}_{6} \mathrm{H}_{3} \mathrm{Cl}-2 ; \mathrm{Br}-4$ & $\mathrm{Br}$ \\
$\mathbf{1 8}$ & 1-naphthyl & $\mathrm{H}$ \\
$\mathbf{1 9}$ & 2-thienyl & $\mathrm{H}$ \\
$\mathbf{2 0}$ & 2-thienyl & $\mathrm{Br}$ \\
$\mathbf{2 1}$ & 3-thienyl-($\left(\mathrm{CH}_{3}\right)_{2}-2,5$ & $\mathrm{H}$ \\
$\mathbf{2 2}$ & 3-thienyl- $\left(\mathrm{CH}_{3}\right)_{2}-2,5$ & $\mathrm{Br}$
\end{tabular}

Scheme 1 
a molecular ion peak $\mathrm{m} / \mathrm{z}$ at $280\left(\mathrm{M}^{+}, 5.70 \%\right)$ with a base peak at $\mathrm{m} / \mathrm{z} 69(100 \%)$, and another significant peaks appeared at $\mathrm{m} / \mathrm{z} 263$ (30\%), 235 (21\%), 190 (12\%). IR spectra of 10-22 exhibited the presence of bands at $1702-1739 \mathrm{~cm}^{-1}$ for $\mathrm{C}=\mathrm{O}$ and at $1584-1604 \mathrm{~cm}^{-1}$ for $\mathrm{C}=\mathrm{N} .{ }^{1} \mathrm{H}$ NMR spectra revealed the presence of a triplet at 1.1-1.6 ppm for $\mathrm{CH}_{3}$ ester and a quartet at 4.2-4.5 ppm for $\mathrm{CH}_{2}$ ester. Mass spectrum of compound 14 showed a molecular ion peak $m / z$ at $431\left(\mathrm{M}^{+}, 9.25 \%\right), 433\left(\mathrm{M}^{+}+2,8.85 \%\right)$ with a base peak at $m / z 76(100 \%)$ in addition to other significant peaks at $\mathrm{m} / \mathrm{z} 396$ (26\%), 368 (18\%), 288 (31\%), 152 (14\%).

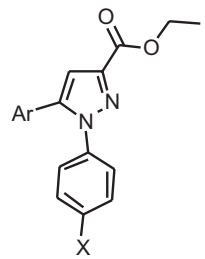

Table I. Physical and analytical data for newly synthesized compounds

\begin{tabular}{|c|c|c|c|c|c|c|c|c|}
\hline \multirow{2}{*}{ Compd. } & \multirow{2}{*}{ Ar } & \multirow{2}{*}{$X$} & \multirow{2}{*}{$\begin{array}{l}\text { Formula } \\
\left(M_{\mathrm{r}}\right)\end{array}$} & \multirow{2}{*}{$\begin{array}{l}\text { M. p. } \\
\left({ }^{\circ} \mathrm{C}\right)\end{array}$} & \multirow{2}{*}{$\begin{array}{l}\text { Yield } \\
(\%)\end{array}$} & \multicolumn{3}{|c|}{ Analysis (calcd./found) (\%) } \\
\hline & & & & & & $\mathrm{C}$ & $\mathrm{H}$ & $\mathrm{N}$ \\
\hline 2 & $\mathrm{C}_{6} \mathrm{H}_{3}\left(\mathrm{OCH}_{3}\right)_{2}-3,4$ & $\mathrm{H}$ & $\begin{array}{l}\mathrm{C}_{14} \mathrm{H}_{16} \mathrm{O}_{6} \\
(280.27)\end{array}$ & $156-157$ & 76 & $59.99 / 59.58$ & $5.75 / 5.49$ & - \\
\hline 3 & $\mathrm{C}_{6} \mathrm{H}_{3}\left(\mathrm{OCH}_{3}\right)_{2}-2,5$ & $\mathrm{H}$ & $\begin{array}{l}\mathrm{C}_{14} \mathrm{H}_{16} \mathrm{O}_{6} \\
(280.27)\end{array}$ & 217-218 & 69 & $59.99 / 59.71$ & $5.75 / 5.98$ & - \\
\hline 4 & $\mathrm{C}_{6} \mathrm{H}_{3} \mathrm{Cl}_{2}-2,5$ & $\mathrm{H}$ & $\begin{array}{l}\mathrm{C}_{12} \mathrm{H}_{10} \mathrm{Cl}_{2} \mathrm{O}_{42} \\
(289.11)\end{array}$ & $78-79$ & 88 & $49.85 / 49.63$ & $3.49 / 3.16$ & - \\
\hline 5 & $\mathrm{C}_{6} \mathrm{H}_{3} \mathrm{Cl}-2, \mathrm{Br}-4$ & $\mathrm{H}$ & $\begin{array}{l}\mathrm{C}_{12} \mathrm{H}_{10} \mathrm{BrClO}_{4} \\
(333.56)\end{array}$ & $90-91$ & 65 & $43.21 / 43.46$ & $3.02 / 3.25$ & - \\
\hline 6 & 1-naphthyl & $\mathrm{H}$ & $\begin{array}{l}\mathrm{C}_{10} \mathrm{H}_{14} \mathrm{O}_{4} \\
(270.28)\end{array}$ & $120-121$ & 69 & $71.10 / 71.32$ & $5.22 / 5.46$ & - \\
\hline 7 & 2-thienyl & $\mathrm{H}$ & $\begin{array}{l}\mathrm{C}_{10} \mathrm{H}_{10} \mathrm{O}_{4} \mathrm{~S} \\
(226.25)\end{array}$ & $86-87$ & 78 & $53.09 / 53.36$ & $4.46 / 4.15$ & - \\
\hline 8 & 3-thienyl-( $\left(\mathrm{CH}_{3}\right)_{2}-2,5$ & $\mathrm{H}$ & $\begin{array}{l}\mathrm{C}_{12} \mathrm{H}_{14} \mathrm{O}_{4} \mathrm{~S} \\
(254.30)\end{array}$ & $208-209$ & 66 & $56.68 / 56.36$ & $5.55 / 4.78$ & - \\
\hline 10 & $\mathrm{C}_{6} \mathrm{H}_{4} \mathrm{Cl}-4$ & $\mathrm{Br}$ & $\begin{array}{l}\mathrm{C}_{18} \mathrm{H}_{14} \mathrm{BrClN}_{2} \mathrm{O}_{2} \\
(403.99)\end{array}$ & $117-118$ & 89 & $53.29 / 53.54$ & $3.48 / 3.72$ & $6.91 / 6.63$ \\
\hline 11 & $\mathrm{C}_{6} \mathrm{H}_{3}\left(\mathrm{OCH}_{3}\right)_{2}-3,4$ & $\mathrm{H}$ & $\begin{array}{l}\mathrm{C}_{20} \mathrm{H}_{20} \mathrm{~N}_{2} \mathrm{O}_{4} \\
(352.38)\end{array}$ & $177-178$ & 88 & $68.17 / 67.82$ & $5.72 / 5.99$ & $7.95 / 8.35$ \\
\hline 12 & $\mathrm{C}_{6} \mathrm{H}_{3}\left(\mathrm{OCH}_{3}\right)_{2}-3,4$ & $\mathrm{Br}$ & $\begin{array}{l}\mathrm{C}_{20} \mathrm{H}_{19} \mathrm{BrN}_{2} \mathrm{O}_{4} \\
(431.28)\end{array}$ & $>300$ & 75 & $55.70 / 55.94$ & $4.44 / 4.31$ & $6.50 / 6.21$ \\
\hline 13 & $\mathrm{C}_{6} \mathrm{H}_{3}\left(\mathrm{OCH}_{3}\right)_{2}-2,5$ & $\mathrm{H}$ & $\begin{array}{l}\mathrm{C}_{20} \mathrm{H}_{20} \mathrm{~N}_{2} \mathrm{O}_{4} \\
(352.38)\end{array}$ & $156-157$ & 75 & $68.17 / 68.51$ & $5.72 / 5.49$ & $7.95 / 7.66$ \\
\hline 14 & $\mathrm{C}_{6} \mathrm{H}_{3}\left(\mathrm{OCH}_{3}\right)_{2}-2,5$ & $\mathrm{Br}$ & $\begin{array}{l}\mathrm{C}_{20} \mathrm{H}_{19} \mathrm{BrN}_{2} \mathrm{O}_{4} \\
(431.28)\end{array}$ & $136-137$ & 68 & $55.70 / 55.45$ & $4.44 / 4.21$ & $6.50 / 6.77$ \\
\hline
\end{tabular}




\begin{tabular}{|c|c|c|c|c|c|c|c|c|}
\hline 15 & $\mathrm{C}_{6} \mathrm{H}_{3} \mathrm{Cl}_{2}-2,5$ & $\mathrm{H}$ & $\begin{array}{l}\mathrm{C}_{18} \mathrm{H}_{14} \mathrm{Cl}_{2} \mathrm{~N}_{2} \mathrm{O}_{2} \\
(361.22)\end{array}$ & 153-154 & 73 & $59.85 / 59.53$ & $3.91 / 3.65$ & 7.76/7.48 \\
\hline 16 & $\mathrm{C}_{6} \mathrm{H}_{3} \mathrm{Cl}-2, \mathrm{Br}-4$ & $\mathrm{H}$ & $\begin{array}{l}\mathrm{C}_{18} \mathrm{H}_{14} \mathrm{BrClN}_{2} \mathrm{O}_{2} \\
(405.67)\end{array}$ & $>300$ & 70 & $53.29 / 53.56$ & $3.48 / 3.18$ & $6.91 / 6.68$ \\
\hline 17 & $\mathrm{C}_{6} \mathrm{H}_{3} \mathrm{Cl}-2, \mathrm{Br}-4$ & $\mathrm{Br}$ & $\begin{array}{l}\mathrm{C}_{18} \mathrm{H}_{13} \mathrm{Br}_{2} \mathrm{ClN}_{2} \mathrm{O}_{2} \\
(481.90)\end{array}$ & $>300$ & 65 & $44.62 / 44.26$ & $2.70 / 2.51$ & $5.78 / 5.47$ \\
\hline 18 & 1-naphthyl & $\mathrm{H}$ & $\begin{array}{l}\mathrm{C}_{22} \mathrm{H}_{18} \mathrm{~N}_{2} \mathrm{O}_{2} \\
(341.39)\end{array}$ & $>300$ & 82 & $77.17 / 77.50$ & $5.30 / 5.12$ & $8.18 / 8.48$ \\
\hline 19 & 2-thienyl & $\mathrm{H}$ & $\begin{array}{l}\mathrm{C}_{16} \mathrm{H}_{14} \mathrm{~N}_{2} \mathrm{O}_{2} \mathrm{~S} \\
(298.36)\end{array}$ & $176-178$ & 78 & $64.41 / 64.12$ & $4.73 / 4.49$ & 9.39/9.65 \\
\hline 20 & 2-thienyl & $\mathrm{Br}$ & $\begin{array}{l}\mathrm{C}_{16} \mathrm{H}_{13} \mathrm{BrN}_{2} \mathrm{O}_{2} \mathrm{~S} \\
(377.26)\end{array}$ & 110-111 & 86 & $50.94 / 50.69$ & $3.47 / 3.72$ & 7.43/7.19 \\
\hline 21 & 3-thienyl- $\left(\mathrm{CH}_{3}\right)_{2}-2,5$ & $\mathrm{H}$ & $\begin{array}{l}\mathrm{C}_{18} \mathrm{H}_{18} \mathrm{~N}_{2} \mathrm{O}_{2} \mathrm{~S} \\
(326.41)\end{array}$ & $168-169$ & 77 & $66.23 / 66.51$ & $5.56 / 5.24$ & $8.58 / 8.91$ \\
\hline 22 & 3-thienyl-( $\left(\mathrm{CH}_{3}\right)_{2}-2,5$ & $\mathrm{Br}$ & $\begin{array}{l}\mathrm{C}_{18} \mathrm{H}_{17} \mathrm{BrN}_{2} \mathrm{O}_{2} \mathrm{~S} \\
(405.31)\end{array}$ & 191-192 & 83 & $53.34 / 53.65$ & $4.23 / 4.00$ & $6.91 / 6.66$ \\
\hline
\end{tabular}

Table II. Spectral characterization of the newly synthesized compounds

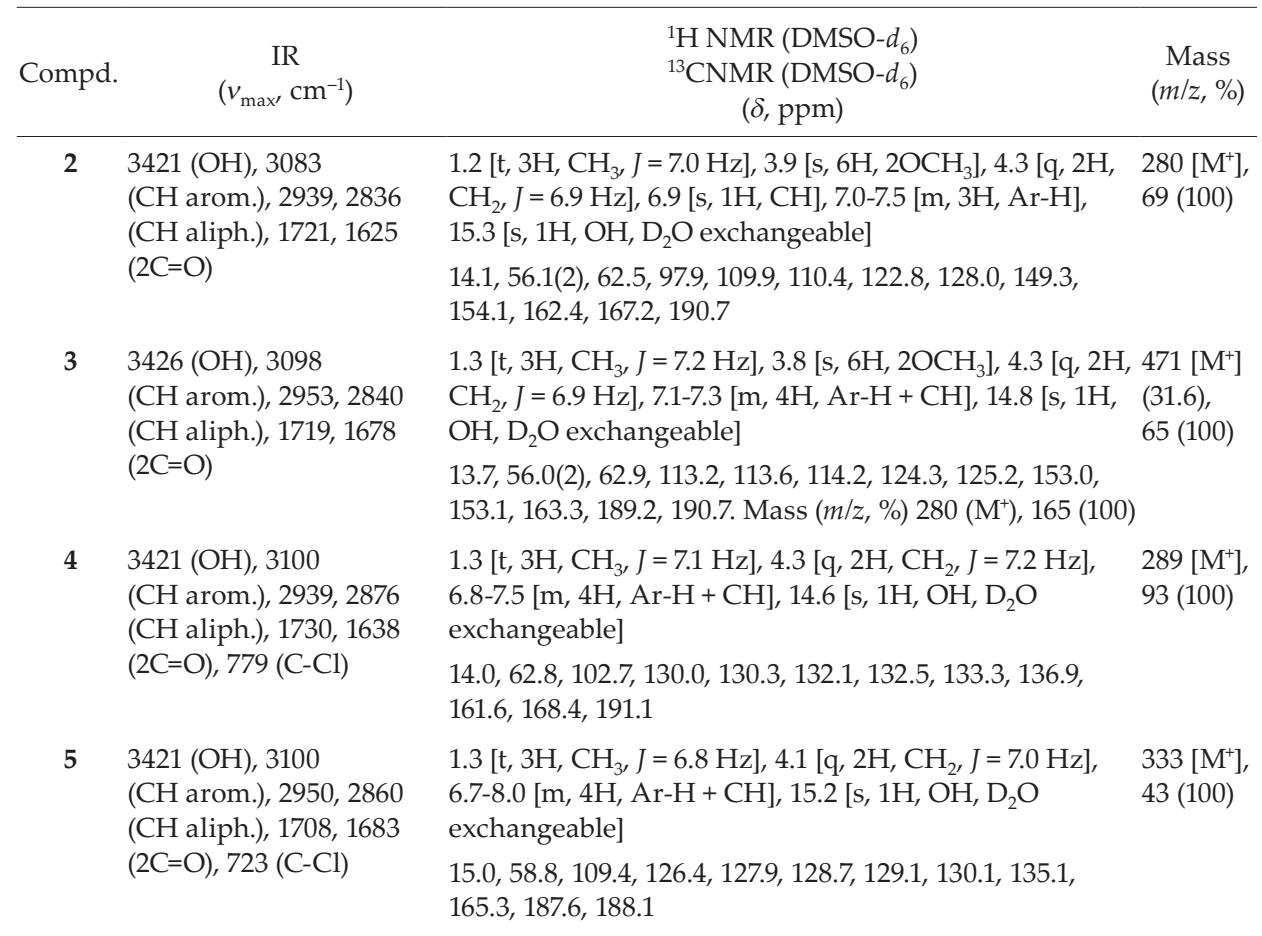


$63446(\mathrm{OH}), 3085$

(CH arom.), 2950, 2825 (CH aliph.), 1743, 1654 $(2 \mathrm{C}=\mathrm{O})$

$73462(\mathrm{OH}), 3110$ (CH arom.), 2963, 2861 (CH aliph.), 1751, 1685 $(2 \mathrm{C}=\mathrm{O})$

$83430(\mathrm{OH}), 3100$ (CH arom.), 2930, 2871 (CH aliph.), 1708, 1641 $(2 \mathrm{C}=\mathrm{O})$

103098 (CH arom.), 2965, 2861 (CH aliph.), 1714, $(\mathrm{C}=\mathrm{O}), 1608(\mathrm{C}=\mathrm{N}), 765$ (C-Cl)

113091 (CH arom.), 2982, 2951 (CH aliph.), 1721, (C=O), 1589 (C=N)

123078 (CH arom.), 2950, 2835 (CH aliph.), 1722, (C=O), $1610(\mathrm{C}=\mathrm{N})$

133100 (CH arom.), 2948, 2835 (CH aliph.), 1739, $(\mathrm{C}=\mathrm{O}), 1584(\mathrm{C}=\mathrm{N})$

143100 (CH arom.), 2978, 2886 (CH aliph.), 1718, (C=O), $1602(\mathrm{C}=\mathrm{N})$

3095 (CH arom.), 2983, 2872 (CH aliph.), 1702, $(\mathrm{C}=\mathrm{O}), 1587(\mathrm{C}=\mathrm{N}), 757$ (C-Cl)

16

3098 (CH arom.), 2970, 2836 (CH aliph.), 1708, (C=O), $1604(\mathrm{C}=\mathrm{N}), 779$ (C-Cl) $1.3\left[\mathrm{t}, 3 \mathrm{H}, \mathrm{CH}_{3}, J=7.1 \mathrm{~Hz}\right], 4.2\left[\mathrm{q}, 2 \mathrm{H}, \mathrm{CH}_{2}, J=6.9 \mathrm{~Hz}\right], \quad 270\left[\mathrm{M}^{+}\right]$ 6.8-8.1 [m, 7H, Ar-H], 8.2 [s, 1H, CH], 15.1 [s, 1H, OH, 54 (100) $\mathrm{D}_{2} \mathrm{O}$ exchangeable]

$13.8,62.2,102.6,123.0,127.1,127.6,128.9,129.8,130.1$, 132.1, 133.3, 134.1, 135.2, 161.7, 190.2, 198.2

$1.3\left[\mathrm{t}, 3 \mathrm{H}, \mathrm{CH}_{3}, \mathrm{~J}=6.9 \mathrm{~Hz}\right], 4.3\left[\mathrm{q}, 2 \mathrm{H}, \mathrm{CH}_{2}, \mathrm{~J}=7.2 \mathrm{~Hz}\right.$, 6.8-7.7 [m, 4H, Ar-H + CH] $14.8\left[\mathrm{~s}, 1 \mathrm{H}, \mathrm{OH}, \mathrm{D}_{2} \mathrm{O}\right.$ exchangeable]

13.7, 62.1, 97.9, 129.1, 130.3, 133.0, 139.0, 161.4, 168.9, 188.8

$1.3\left[\mathrm{t}, 3 \mathrm{H}, \mathrm{CH}_{3}, J=7.3 \mathrm{~Hz}\right], 2.3\left[\mathrm{~s}, 6 \mathrm{H}, 2 \mathrm{CH}_{3}\right], 4.4\left[\mathrm{q}, 2 \mathrm{H}, 254\left[\mathrm{M}^{+}\right]\right.$, $\left.\mathrm{CH}_{2}, \mathrm{~J}=7.1 \mathrm{~Hz}\right], 6.6-8.0[\mathrm{~m}, 2 \mathrm{H}, \mathrm{Ar}-\mathrm{H}+\mathrm{CH}], 11.4[\mathrm{~s}, 1 \mathrm{H}, 43$ (100) $\mathrm{OH}, \mathrm{D}_{2} \mathrm{O}$ exchangeable]

$10.0,13.8,14.3,61.3,109.5,123.0,135.1,143.5,155.2,160.4$, $186.1,188.3$

$1.3\left[\mathrm{t}, 3 \mathrm{H}, \mathrm{CH}_{3}, J=7.6 \mathrm{~Hz}\right], 4.1\left[\mathrm{q}, 2 \mathrm{H}, \mathrm{CH}_{2}, J=7.5 \mathrm{~Hz}\right], \quad 403\left[\mathrm{M}^{+}\right]$ 6.9-8.1 [m, 9H, Ar-H + CH pyrazole] $78(100)$

$14.0,63.2,115.2,120.8(2), 126.1,126.9$ (2), 127.7, 128.6, 129.6 (2), 130.6, 133.5, 138.1, 141.0, 144.1, 163.5

$1.1\left[\mathrm{t}, 3 \mathrm{H}, \mathrm{CH}_{3}, J=7.6 \mathrm{~Hz}\right], 3.6\left[\mathrm{~s}, 6 \mathrm{H}, 2 \mathrm{OCH}_{3}\right], 4.2\left[\mathrm{q}, 2 \mathrm{H}, 352\left[\mathrm{M}^{+}\right]\right.$, $\left.\mathrm{CH}_{2}, \mathrm{~J}=7.4 \mathrm{~Hz}\right], 6.9-8.0[\mathrm{~m}, 9 \mathrm{H}, \mathrm{Ar}-\mathrm{H}+\mathrm{CH}$ pyrazole $] \quad 77(100)$

$12.5,56.4,55.8,63.2,110.1,112.1,115.2,116.8,118.1,123.1$ (2), 126.2, 128.6 (2), 140.4, 140.6, 146.8, 150.6, 151.3, 165.7

$1.4\left[\mathrm{t}, 3 \mathrm{H}, \mathrm{CH}_{3} J=7.3 \mathrm{~Hz}\right], 3.5\left[\mathrm{~s}, 6 \mathrm{H}, 2 \mathrm{OCH}_{3}\right], 4.4\left[\mathrm{q}, 2 \mathrm{H}, 431\left(\mathrm{M}^{+}\right)\right.$, $\left.\mathrm{CH}_{2}, J=7.3 \mathrm{~Hz}\right], 6.9-8.1[\mathrm{~m}, 9 \mathrm{H}, \mathrm{Ar}-\mathrm{H}+\mathrm{CH}$ pyrazole $] \quad 42(100)$

$14.2,56.2,56.3,62.4,111.6,112.6,114.6,118.3,119.7,122.1$, 126.3 (2), 127.5 (2), 139.7, 139.8, 144.6, 151.5, 153.1, 164.2

$1.3\left[\mathrm{t}, 3 \mathrm{H}, \mathrm{CH}_{3}, \mathrm{~J}=7.1 \mathrm{~Hz}\right], 3.8\left[\mathrm{~s}, 6 \mathrm{H}, 2 \mathrm{OCH}_{3}\right], 4.4\left[\mathrm{q}, 2 \mathrm{H}, 352\left[\mathrm{M}^{+}\right]\right.$, $\left.\mathrm{CH}_{2}, \mathrm{~J}=7.2 \mathrm{~Hz}\right], 6.6-7.8[\mathrm{~m}, 9 \mathrm{H}, \mathrm{Ar}-\mathrm{H}+\mathrm{CH}$ pyrazole $] 59(100)$

$13.8,55.2,55.3,62.9,110.7,111.5,115.8,116.3,119.0,123.7$ (2), 127.8, 128.4 (2), 140.0, 140.9, 144.2, 150.1, 152.8, 163.1

$1.1\left[\mathrm{t}, 3 \mathrm{H}, \mathrm{CH}_{3}, J=7.5 \mathrm{~Hz}\right], 3.7\left[\mathrm{~s}, 6 \mathrm{H}, 2 \mathrm{OCH}_{3}\right], 4.5\left[\mathrm{q}, 2 \mathrm{H}, 431\left[\mathrm{M}^{+}\right]\right.$, $\left.\mathrm{CH}_{2}, \mathrm{~J}=7.4 \mathrm{~Hz}\right], 6.7-7.5[\mathrm{~m}, 9 \mathrm{H}, \mathrm{Ar}-\mathrm{H}+\mathrm{CH}$ pyrazole $] \quad 76(100)$

$13.8,55.2,55.3,62.9,110.7,112.8,115.8,116.7,119.0,123.7$, 127.8 (2), 128.4 (2), 140.0, 140.9, 144.2, 150.1, 152.8, 163.1

$1.2\left[\mathrm{t}, 3 \mathrm{H}, \mathrm{CH}_{3}, \mathrm{~J}=7.2 \mathrm{~Hz}\right], 4.2\left[\mathrm{q}, 2 \mathrm{H}, \mathrm{CH}_{2}, J=7.1 \mathrm{~Hz}\right]$, 6.8-8.0 [m, 9H, Ar-H + CH pyrazole]

$361\left[\mathrm{M}^{+}\right]$ 77 (100)

14.2, 62.9, 111.5, 121.7 (2), 124.3, 128.5, 129.5 (2), 130.2, 130.4, 131.1, 131.8, 132.6, 136.4, 140.4, 143.4, 161.3

$1.1\left[\mathrm{t}, 3 \mathrm{H}, \mathrm{CH}_{3}, J=7.4 \mathrm{~Hz}\right], 4.4\left[\mathrm{q}, 2 \mathrm{H}, \mathrm{CH}_{2}, J=7.4 \mathrm{~Hz}\right]$, 7.5-8.2 [m, 9H, Ar-H + CH pyrazole]

$405\left[\mathrm{M}^{+}\right]$, $46(100)$ 133.5, 133.6, 134.3, 134.6, 135.3, 145.7, 146.4, 162.1 
173060 (CH arom.), 2955, 2866 (CH aliph.), 1704, $(\mathrm{C}=\mathrm{O}), 1610(\mathrm{C}=\mathrm{N}), 850$ (C-Cl)

183053 (CH arom.), 2926, 2843 (CH aliph.), 1700, (C=O), $1570(\mathrm{C}=\mathrm{N})$

193100 (CH arom.), 2977, 2862 (CH aliph.), 1728, $(\mathrm{C}=\mathrm{O}), 1599(\mathrm{C}=\mathrm{N})$

203099 (CH arom.), 2981, 2886 (CH aliph.), 1709, $(\mathrm{C}=\mathrm{O}), 1590(\mathrm{C}=\mathrm{N})$

213085 (CH arom.), 2968, 2840 (CH aliph.), 1710, $(\mathrm{C}=\mathrm{O}), 1560(\mathrm{C}=\mathrm{N})$

223098 (CH arom.), 2949, 2871 (CH aliph.), 1708, (C=O), 1599 (C=N) $1.2\left[\mathrm{t}, 3 \mathrm{H}, \mathrm{CH}_{3}, \mathrm{~J}=7.3 \mathrm{~Hz}\right], 4.3\left[\mathrm{q}, 2 \mathrm{H}, \mathrm{CH}_{2}, J=7.4 \mathrm{~Hz}\right]$, 6.5-7.9 [m, 8H, Ar-H + CH pyrazole]

$481\left[\mathrm{M}^{+}\right]$ $43(100)$

13.6, 62.4, 109.3, 120.3, 123.0 (2), 124.5, 128.4, 130.6, 131.0, $134.0,134.5,135.1,136.7,138.2,144.1,144.5,160.3$

$1.2\left[\mathrm{t}, 3 \mathrm{H}, \mathrm{CH}_{3}, \mathrm{~J}=7.3 \mathrm{~Hz}\right], 4.3\left[\mathrm{q}, 2 \mathrm{H}, \mathrm{CH}_{2}, J=7.3 \mathrm{~Hz}\right]$,

6.9-8.0 [m, 13H, Ar- $\mathrm{H}+\mathrm{CH}$ pyrazole $]$

$342\left[\mathrm{M}^{+}\right]$, $129(100)$

$13.8,62.1,102.6,123.0$ (2), 124.9, 126.6 (2), 127.1, 127.7, 128.7 (2), 128.8, 129.8 (2), 130.1, 132.2, 133.2, 135.4, 148.6, $148.9,161.8$

$1.2\left[\mathrm{t}, 3 \mathrm{H}, \mathrm{CH}_{3}, \mathrm{~J}=7.5 \mathrm{~Hz}\right], 4.3\left[\mathrm{q}, 2 \mathrm{H}, \mathrm{CH}_{2}, J=7.3 \mathrm{~Hz}\right]$, 6.4-7.9 [m, 9H, Ar-H + CH pyrazole]

$298\left[\mathrm{M}^{+}\right]$ 77 (100)

13.7, 62.1, 111.6, 119.6, 121.4 (2), 128.2, 128.8, 129.1, 129.7, 131.9 (2), 138.5, 140.0, 145.3, 163.4

$1.2\left[\mathrm{t}, 3 \mathrm{H}, \mathrm{CH}_{3}, \mathrm{~J}=7.2 \mathrm{~Hz}\right], 4.3\left[\mathrm{q}, 2 \mathrm{H}, \mathrm{CH}_{2}, J=7.2 \mathrm{~Hz}\right]$, 7.0-7.7 [m, 8H, Ar-H + CH pyrazole]

$377\left[\mathrm{M}^{+}\right]$ $76(100)$

14.1, 60.6, 109.0, 122.7, 127.7 (2), 128.6, 128.7, 128.8, 131.5, 132.3 (2), 137.9, 138.7, 143.4, 161.7

$1.1\left[\mathrm{t}, 3 \mathrm{H}, \mathrm{CH}_{3}, J=7.4 \mathrm{~Hz}\right], 2.5\left[\mathrm{~s}, 6 \mathrm{H}, 2 \mathrm{CH}_{3}\right], 4.4[\mathrm{q}, 2 \mathrm{H}$, $\mathrm{CH}_{2}, \mathrm{~J}=7.5 \mathrm{~Hz}$ ], 6.8-7.9 [m, 7H, Ar-H + CH pyrazole]

$326\left[\mathrm{M}^{+}\right]$ $91(100)$

11.6, 14.5, 14.8, 62.6, 109.4, 120.8 (2), 123.0, 126.5, 127.1, 128.0 (2), 134.5, 135.2, 136.8, 139.3, 144.4, 160.4

$1.6\left[\mathrm{t}, 3 \mathrm{H}, \mathrm{CH}_{3}, J=7.1 \mathrm{~Hz}\right], 2.6\left[\mathrm{~s}, 6 \mathrm{H}, 2 \mathrm{CH}_{3}\right], 4.5[\mathrm{q}, 2 \mathrm{H}$, $\left.\mathrm{CH}_{2}, \mathrm{~J}=7.1 \mathrm{~Hz}\right], 6.5-7.9[\mathrm{~m}, 6 \mathrm{H}, \mathrm{Ar}-\mathrm{H}+\mathrm{CH}$ pyrazole $]$

$405\left[\mathrm{M}^{+}\right]$, $155(100)$ 128.8 (2), 130.3, 135.2, 136.9, 139.9, 143.5, 160.5

\section{Antimicrobial screening}

Table III lists the results of the antimicrobial screening of the new compounds against some Gram-positive and some Gram-negative bacteria and fungi. The antibacterial screening revealed that compound $21\left(M I C_{E \text {. coli }}=0.038 \mu \mathrm{mol} \mathrm{mL}^{-1}, M_{I} C_{P \text {. aeruginosa }}=0.076 \mu \mathrm{mol}\right.$ $\left.\mathrm{mL}^{-1}\right)$ is nearly as active as ampicillin $\left(M I C_{E \text {. coli }}=0.033, M I C_{P \text {. aeruginosa }} 0.067 \mu \mathrm{mol} \mathrm{mL}^{-1}\right)$. The antifungal screening showed that compound $16\left(\mathrm{MIC}=0.015 \mu \mathrm{mol} \mathrm{mL} \mathrm{m}^{-1}\right)$ exhibited more potency activity than fluconazole $\left(0.020 \mu \mathrm{mol} \mathrm{mL} \mathrm{m}^{-1}\right)$ against $C$. parapsilosis. Also, compound 16 showed lower activity than fluconazole $\left(0.005 \mu \mathrm{mol} \mathrm{mL}^{-1}\right)$ against $C$. albicans and $C$. tropicalis $\left(M I C_{C \text {. albicans }}=0.015 \mu \mathrm{mol} \mathrm{mL}{ }^{-1}, M I C_{C \text {. tropicalis }}=0.030 \mu \mathrm{mol} \mathrm{mL}^{-1}\right)$. From these results it was found that the presence of 2,5-dimethylthienyl moiety at 5-position and unsubsituted phenyl ring at 1-position in compound 21 enhanced the antibacterial activity against $E$. coli, while the presence of (un)substituted aromatic hydrocarbon moiety or unsubstituted thienyl at 5-position and (un)substituted phenyl ring at 1-position in compounds 9-20, 22 revealed a moderate or weak activity. Dihalogenated phenyl at 5-position and unsubstituted phenyl at 1-position in compound $\mathbf{1 6}$ revealed significant activity compared with fluconazole as reference drug against $C$. parapsilosis. The remaining compounds were found either weak or inactive. 
A. A. Radwan et al.: Novel ethyl 1,5-disubstituted-1H-pyrazole-3-carboxylates as a new class of antimicrobial agents, Acta Pharm. 64 (2014) 335-344

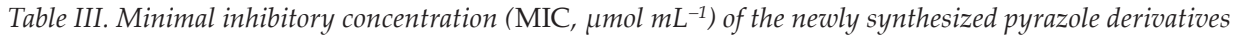

\begin{tabular}{cccccccc}
\hline Compd. & $\begin{array}{c}\text { Bacillus } \\
\text { subtilis } \\
\text { (NCTC }\end{array}$ & $\begin{array}{c}\text { Staphylococcus } \\
\text { aureus } \\
\text { (NCTC- }\end{array}$ & $\begin{array}{c}\text { E. coli } \\
\text { (NCTC }\end{array}$ & $\begin{array}{c}\text { Pseudomonas } \\
\text { aeruginosa } \\
\text { (ATCC }\end{array}$ & $\begin{array}{c}\text { Candida } \\
\text { albicans } \\
\text { (ATCC- }\end{array}$ & $\begin{array}{c}\text { Candida } \\
\text { tropicalis } \\
\text { (ATCC- }\end{array}$ & $\begin{array}{c}\text { Candida } \\
\text { parapsilosis } \\
\text { (ATCC- }\end{array}$ \\
\hline $\mathbf{1 0 4 0 )}$ & $7447)$ & $10416)$ & $10145)$ & $10231)$ & $66029)$ & $22019)$ \\
\hline $\mathbf{1 0}$ & 0.306 & 0.153 & 0.153 & 0.306 & 0.153 & 0.153 & 0.306 \\
$\mathbf{1 1}$ & 0.124 & 0.248 & 0.124 & 0.248 & 0.124 & 0.062 & 0.124 \\
$\mathbf{1 2}$ & 0.142 & 0.142 & 0.142 & 0.142 & 0.071 & 0.142 & 0.142 \\
$\mathbf{1 3}$ & 0.232 & 0.116 & 0.232 & 0.232 & 0.058 & 0.116 & 0.116 \\
$\mathbf{1 4}$ & 0.142 & 0.142 & 0.142 & 0.142 & 0.284 & $\mathrm{NA}^{*}$ & NA \\
$\mathbf{1 5}$ & 0.116 & 0.232 & 0.116 & 0.116 & 0.232 & $\mathrm{NA}$ & $\mathrm{NA}$ \\
$\mathbf{1 6}$ & 0.138 & 0.138 & 0.138 & 0.138 & 0.069 & 0.069 & 0.034 \\
$\mathbf{1 7}$ & 0.246 & 0.123 & 0.123 & 0.246 & 0.015 & 0.030 & 0.015 \\
$\mathbf{1 8}$ & 0.103 & 0.103 & 0.103 & 0.103 & $\mathrm{NA}$ & $\mathrm{NA}$ & NA \\
$\mathbf{1 9}$ & 0.293 & $\mathrm{NA}$ & 0.146 & $\mathrm{NA}$ & 0.293 & 0.293 & 0.293 \\
$\mathbf{2 0}$ & $\mathrm{NA}$ & 0.167 & $\mathrm{NA}$ & 0.325 & 0.167 & 0.167 & 0.167 \\
$\mathbf{2 1}$ & $\mathrm{NA}$ & $\mathrm{NA}$ & 0.265 & $\mathrm{NA}$ & 0.132 & 0.132 & 0.132 \\
$\mathbf{2 2}$ & 0.076 & 0.076 & 0.038 & 0.076 & $\mathrm{NA}$ & 0.153 & NA \\
Ampicillin & 0.123 & 0.246 & 0.123 & 0.123 & 0.061 & 0.061 & NA \\
Fluconazole & 0.033 & 0.008 & 0.033 & 0.067 & $\mathrm{NA}$ & 0.067 & 0.134 \\
\hline
\end{tabular}

NA - Compounds having MIC $>0.5 \mu \mathrm{mol} \mathrm{mL} \mathrm{m}^{-1}$.

\section{CONCLUSIONS}

This study showed that the newly synthesized ethyl 5-(2,5-dimethylthiophen-3-yl)-1phenyl-1H-pyrazole-3-carboxylate (21) was found to be of comparable activity as ampicillin against E. coli and P. aeruginosa. The same is true for ethyl 5-(4-bromo-2-chlorophenyl)1-phenyl-1H-pyrazole-3-carboxylate (16) vs. fluconazole against Candida parapsilosis. This is due to the presence of dihalogenated phenyl at 5-position and unsubstituted phenyl at 1-position of the pyrazole ring.

Acknowledgements. - The authors are grateful to the sponsorship and of the Research Center, College of Pharmacy and the Deanship of Scientific Research, King Saud University, Riyadh, Saudi Arabia. IR and NMR spectra were recorded in the same research centre. Antimicrobial screenings researches were done in the central laboratory of microbiology of the same University. 


\section{REFERENCES}

1. N. D. Gaikwad, S. V. Patil and V. D. Bobade, Synthesis and antimicrobial activity of novel thiazole substituted pyrazole derivatives, J. Het. Chem. 50 (2013) 519-527; DOI: 10.1002/jhet.1513.

2. M. S. Essam and M. M. H Nagwa, Synthesis and antimicrobial evaluation of some pyrazole derivatives, Molecules 17 (2012) 4962-4971; DOI: 10.3390/molecules17054962.

3. Z. Chuan-Yu, L. Xing-Hai, W. Bao-Lei, W. Su-Hua and L. Zheng-Ming, Synthesis and antifungal activities of new pyrazole derivatives via 1,3-dipolar cycloaddition reaction, Chem. Biol. Drug Des. 75 (2010) 489-493; DOI: 10.1111/j.1747-0285.2010.00948.x.

4. E. M. Flefel, W. A. Tantawy, W. A. El-Sayed, H. H. Sayed and N. M. Fathy, Synthesis and anticancer activity of new substituted pyrazoles and their derived 1,2,4-triazoles and sugar derivatives, J. Het. Chem. 50 (2013) 344-350; DOI: 10.1002/jhet.1122.

5. S. M. El-Moghazy, F. F. Barsoum, H. M. Abdel-Rahman and A. A. Marzouk, Synthesis and antiinflammatory activity of some pyrazole derivatives, Med. Chem. Res. 21 (2012) 1722-1733; DOI: 10.1007/s00044-011-9691-4.

6. M. M. Ghorab, F. A. Ragab, S. I. Alqasoumi, A. M. Alafeefy and S. A. Aboulmagd, Synthesis of some new pyrazolo[3,4-d]pyrimidine derivatives of expected anticancer and radioprotective activity, Eur. J. Med. Chem. 45 (2010) 171-178; DOI: 10.1016/j.bmc.2013.11.042.

7. B. Parashar, A. Jain, S. Bharadwaj and V. K. Sharma, Synthesis and pharmacological properties of some novel pyrazolidine and pyrazole derivatives, Med. Chem. Res. 21 (2012) 1692-1699; DOI: 10.1007/s00044-011-9687-0.

8. A. B. Adnan, H. Ariaya, A. Henok and E. A. B. Alaa, Synthesis and biological evaluation of some pyrazole derivatives as anti-malarial agents, Arch. Pharm. Chem. Life Sci. 345 (2012) 147-154; DOI: 10.1002/ardp.201100078.

9. M. M. Ghorab, H. I. Zienab, A. Mohamad and A. A. Radwan, Synthesis, antimicrobial evaluation and molecular modelling of novel sulfonamides carrying a biologically active quinazoline nucleus, J. Pharm. Res. 36 (2013) 660-670; DOI: 10.1007/s12272-013-0094-6.

10. M. M. Ghorab, H. I. Zienab, A. A. Radwan and A. Mohamad, Synthesis and pharmacophore modeling of novel quinazolines bearing a biologically active sulfonamide moiety, Acta Pharm. 63 (2013) 1-18; DOI: 10.2478/acph-2013-0006.

11. M. Lindler, W. Sippl and A. A. Radwan, Pharmacophore elucidation and molecular docking studies on 5-phenyl-1-(3-pyridyl)-1H-1,2,4-triazole-3-carboxylic acid derivatives as COX-2 inhibitors, Sci. Pharm. 78 (2010) 195-214; DOI: 10.3797/scipharm.0912-19.

12. M. M. Ghorab, F. A. Ragab, H. I. Heiba and M. G. El-Gazzar, Synthesis, in vitro anticancer screening and radiosensitizing evaluation of some new 4-[3-(substituted)thioureido]- $N$-(quinoxalin-2-yl)benzenesulfonamide derivatives, Acta Pharm. 61 (2011) 415-425; DOI: 10.2478/v10007-011-0040-4.

13. M. M. Ghorab, F. A. Ragab, H. I. Hieba, H. A. Yousef and M. G. El-Gazzar, Synthesis of novel pyrazole and pyrimidine derivatives bearing sulfonamide moiety as antitumor and radiosensitizing agents, Med. Chem. Res. 21 (2012) 1376-1383; DOI 10.1007/s00044-013-0721-2.

14. M. S. Al-Dosari, M. M. Ghorab, M. S. Alsaid, Y. M. Nissan and A. B. Ahmed, Synthesis and anticancer activity of some novel trifluoromethylquinolines carrying a biologically active benzenesulfonamide moiety, Eur. J. Med. Chem. 69 (2013) 373-383; DOI: 10.1016/j.ejmech.2013.08.048.

15. A. M. Ali, G. E. Saber, N. M. Mahfouz, M. A. El-Gendy, A. A. Radwan and M. A. E. Hamid, Synthesis and three-dimensional qualitative structure selectivity relationship of 3,5-disubstituted2,4-thiazolidinedion derivatives as COX2 inhibitors, Arch. Pharm. Res. 30 (2007) 1186-1204; DOI: 10.1007/BF02980259.

16. M. M. Ghorab and M. S. Alsaid, Synthesis and antitumor activity of some novel hydrazide, 1,2-dihydropyridine, chromene, and benzochromene derivatives, J. Heterocyclic Chem. 49 (2012) 272-280; DOI: $10.1002 /$ jhet.829. 
17. M. M. Ghorab, F. A. Ragab, H. I. Hieba and W. M. Ghorab, Design and synthesis of some novel quinoline derivatives as anticancer and radiosensitizing agents targeting VEGFR tyrosine kinase, J. Heterocyclic Chem. 48 (2011) 1269-1279; DOI: /10.1002/jhet.749.

18. M. M. Ghorab, M. S. Alsaid and E. M. El-Hossary, In vitro cytotoxic evaluation of some new heterocyclic sulfonamide derivatives, J. Heterocyclic Chem. 48 (2011) 563-571; DOI: 10.1002/jhet.619.

19. M. Alvarado, G. Pilar, M. Macías-González, F. J. Pavón, A. Serrano, N. Jagerovic, J. Elguero, A. Gutiérrez-Rodríguez, S. García-Granda, M. Suardíaz and F. R. de Fonseca, Antiobesity designed multiple ligands: Synthesis of pyrazole fatty acid amides and evaluation as hypophagic agents, Bioorg. Med. Chem. 16 (2008) 10098-10105; DOI: 10.1016/j.bmc.2008.10.023.

20. I. L. Finar and R. J. Hurlock, The skraup reaction with aminopyrazoles, J. Chem. Soc. 1958, 32593263; DOI: 10.1039/JR9580003259.

21. H. Naeimi, Z. S. Nazifi, S. M. Amininezhad and M. Amouheidari, Synthesis, characterization and in vitro antimicrobial activity of some new Schiff bases and their complexes, J. Antibiot. 66 (2013) 687-689; DOI: 10.1038/ja.2013.73. 